\title{
Genetic Counseling for BRCA1/2: \\ A Randomized Controlled Trial of Two Strategies to Facilitate the Education and Counseling Process
}

\author{
Catharine Wang, ${ }^{1,4 *}$ Richard Gonzalez, ${ }^{2}$ Kara J. Milliron, ${ }^{3,5}$ Victor J. Strecher, ${ }^{1,5}$ and Sofia D. Merajver ${ }^{3,5}$ \\ ${ }^{1}$ Department of Health Behavior and Health Education, University of Michigan, School of Public Health, Ann Arbor, Michigan \\ ${ }^{2}$ Department of Psychology, University of Michigan, Ann Arbor, Michigan \\ ${ }^{3}$ Department of Internal Medicine, University of Michigan, Ann Arbor, Michigan \\ ${ }^{4}$ Michigan Center for Genomics and Public Health, University of Michigan, School of Public Health, Ann Arbor, Michigan \\ ${ }^{5}$ Comprehensive Cancer Center, University of Michigan, Ann Arbor, Michigan
}

\begin{abstract}
Due to the complexity of information surrounding $B R C A 1 / 2$ counseling and testing and its time consuming nature, efforts to facilitate the genetic counseling and education process are needed. Using a $2 \times 2$ factorial design, two strategies were examined: a CD-ROM program for patients and a feedback checklist to the genetic counselor on patients' prior misconceptions. A total of 197 women attending a breast and ovarian cancer risk evaluation clinic for $B R C A 1 / 2$ counseling were randomized into one of four conditions: standard care, CD-ROM only, feedback to counselor only, and both CD-ROM and feedback. Counseling outcomes included face-to-face time with the genetics team, knowledge acquisition, changes in worry about having a gene mutation, and genetic testing decisions. Overall, women who viewed the CDROM spent less time with the genetic counselor and were less likely to undergo genetic testing compared to women who did not view the CDROM. Feedback to the genetic counselor resulted in greater gains in knowledge of genetics and breast cancer. Among women less worried at baseline, those who viewed the CD-ROM showed no changes in worry following genetic counseling, in contrast to those who did not view the CD-ROM who increased in worry over time. This latter finding raises concerns about the impact of the increased worry on genetic testing decisions. No interaction effects of the two intervention arms were found. The study results support the importance of both
\end{abstract}

This article contains supplementary material, which may be viewed at the American Journal of Medical Genetics website at http://www.interscience.wiley.com/jpages/1552-4825/suppmat/ index.html.

Grant sponsor: Canadian Institutes of Health Research (CIHR); Grant sponsor: American Association of University Women Educational Foundation (AAUW); Grant sponsor: Susan G. Komen Breast Cancer Foundation; Grant sponsor: Venture Investment Fund of the University of Michigan Health System; Grant sponsor: Blodgett-Butterworth Research Foundation; Grant sponsor: US Army; Grant number: DAMD17-96-1-6255.

*Correspondence to: Catharine Wang, Ph.D., Michigan Center for Genomics and Public Health, University of Michigan School of Public Health, 611 Church Street, Rm. 262, Ann Arbor, MI 481043028. E-mail: clwang@umich.edu

Received 19 July 2004; Accepted 10 November 2004

DOI 10.1002/ajmg.a.30577 strategies as valuable supplements to clinical BRCA1/2 counseling. @ 2005 Wiley-Liss, Inc.

KEY WORDS: genetic counseling; $B R C A 1 / 2$; CDROM; feedback prompts; patient education; counseling outcomes

\section{INTRODUCTION}

The isolation of two genes, BRCA1 in 1994 [Miki et al., 1994] and BRCA2 in 1995 [Wooster et al., 1995], has resulted in the availability of genetic testing for mutations in these genes that cause hereditary breast and ovarian cancer. Prior to undergoing genetic testing, it is considered essential that women are educated and counseled about the benefits, risks, and limitations of testing [Geller et al., 1997; Schneider, 1997]. Providing this information, however, is inherently complex for several reasons. First, the occurrence of breast and/or ovarian cancer is not due to the effects of a single gene, but rather to a complex interaction of many genes and environmental conditions [Guttmacher et al., 2001]. As such, because the risk for these cancers cannot solely be attributed to genetic causes, the interpretation of test results is more challenging. For example, testing positive for a BRCA1/2 mutation does not imply that cancer will inevitably result. Thus, not all individuals who are BRCA1/2 mutation carriers will develop breast or ovarian cancer. Second, testing negative for a $B R C A 1 / 2$ mutation does not preclude a woman from developing cancer because the majority of cancers are sporadic and result from other multifactorial causes. Receiving a negative test result in this case does not offer a complete sense of reassurance (i.e., that the disease will not develop) as it would in the case of genetic testing for single-gene syndromes, such as Huntington disease.

This complexity in testing for BRCA1/2 has been reflected in the amount of time counselors spend with their patients. Counseling sessions for hereditary cancer syndromes have been reported to take longer (up to $2 \mathrm{hr}$ in duration) and require more follow-up sessions compared to counseling for other conditions [Schneider and Marnane, 1997]. The detail contained in these sessions is considered essential for informed consent and in accordance with recommended guidelines [Geller et al., 1997]. Nonetheless, as a result of this complexity, it is not surprising that women have difficulty understanding genetic information [Tessaro et al., 1997]. This raises concerns about the extent to which women are making informed decisions about genetic testing.

As a result of these concerns, it is critical to identify effective intervention strategies to facilitate the genetic counseling and education process. These strategies can target and affect the patient directly or indirectly by targeting the genetic counselor. In part, developing new methods to facilitate genetic counseling could help deal with the resource intensive nature of 
counseling by saving time and ameliorating the strain on genetic counselors to meet the increasing demand for genetic services [Green and Fost, 1997]. More importantly, these strategies can work to improve the outcomes of genetic counseling that are deemed as critical to its success; namely, improving knowledge, providing support, and facilitating decision making [Wang et al., 2004].

\section{Patient-Oriented Strategies}

Strategies to facilitate genetic counseling and education can either be patient- or counselor-oriented. Efforts in recent years on patient-oriented strategies have focused on employing education aids for patients to help enhance the learning and understanding of genetic information. For example, Cull et al. [1998] examined the value of providing an educational videotape either before or following genetic counseling for breast cancer risk. The videotape reviewed information on the genetic basis of breast cancer and strategies to manage risk (e.g., mammography screening). Overall, women who saw the videotape prior to counseling had shorter consultations with the breast cancer surgeon compared to women who saw the video following counseling. Moreover, although there were no differences in perceptions of risk between the two conditions, women in the video-before group had greater knowledge scores immediately following counseling and were more satisfied with the information provided in clinic than women in the videoafter group.

Others have used interactive multimedia to facilate the education process. For example, a CD-ROM computer program was demonstrated to be as effective at educating women about hereditary breast cancer compared to a genetic counselor [Green et al., 2001a, 2004]. Moreover, evaluations of the program by the same group found that women were satisfied with using the computer program as a form of education and found it to be informative, private, a good use of time, and used without causing embarrassment [Green et al., 2001b]. Testing intentions declined following genetic counseling, however, no differences were observed between women who viewed the CDROM in conjuction with genetic counseling compared to genetic counseling alone [Green et al., 2001a]. The extent to which the CD-ROM saved time and allowed the genetic counselor to focus on more relevant psychosocial issues is unknown.

The use of tailored print material to inform women of their risks has also been a recent strategy employed to help faciliate the education process. Women who were at elevated risk were randomized to receive either generic print information on breast cancer risk or tailored print information, which was based on content including their personal medical history, demographics, and preference for information (e.g., more vs. less detail, verbal vs. numeric/graphic) [Skinner et al., 2002]. Women who received the tailored print materials were more knowledgeable about breast cancer and genetics, as well as more accurate in their personal risk estimates. There were no differences in anxiety between tailored and generic print material. Moreover, because the study did not report on whether these women subsequently took part in genetic counseling, it is unclear to what extent these materials would have facilitated the counseling process.

\section{Counselor-Oriented Strategies}

Less has been done to influence patient outcomes by focusing on the genetic counselor as the intervention target. Interventions that have been conducted center on how counseling itself is delivered. For example, group discussions have been employed to disseminate information about personal risk and cancer genetics [Esplen et al., 1998, 2000]. Others have examined how the content of information covered during genetic counseling (i.e., education alone vs. education plus psychosocial counseling) may influence counseling outcomes [Lerman et al., 1997].

In research areas outside of genetic counseling, interventions that target the provider's behavior have been implemented with moderate levels of success. For example, provider or chart prompts such as reminder systems, checklists, and other office systems, have been employed to improve physician breast cancer screening practices [Lane et al., 2001; Engelman et al., 2004], hypertension control [Dickinson et al., 1981], pediatric injury prevention [Barrios et al., 2001], and other preventive behaviors [Tierney et al., 1986; Bordley et al., 2001]. These strategies may provide a mechanism by which to assist genetic counselors in performing their tasks. Strategies such as feedback prompts may prove especially useful at highlighting areas that are important and relevant for individuals such that care is personalized to best meet their needs. In turn, this may have an impact on patient outcomes such as knowledge acquisition.

\section{Present Study}

Several research questions were proposed in efforts to identify strategies to facilitate the genetic counseling process. First, would it be useful to provide pre-counseling educational aids for patients to review prior to seeing the genetic counselor? Second, would attempts to personalize and tailor genetic counseling to the informational needs of the patients (i.e., focused on misconceptions and less on information already understood by patients) improve the interaction between counselor and patient and streamline the education component of the session? Third, would the combination of these approaches work synergistically to produce an effect?

The present study used a $2 \times 2$ factorial design to examine two strategies to facilitate the counseling process: a CD-ROM computer program for patients and a feedback checklist for the genetic counselor. Incorporating this design allowed testing of both main and interaction effects of the two approaches.

A CD-ROM computer program on cancer and genetics was provided to patients prior to the start of the counseling session. As demonstrated by others, use of interactive multimedia has previously been found to be helpful in educating patients on genetics and cancer [Green et al., 2001a] and may be a practical mechanism by which to enable the genetic counselor to focus on more relevant counseling issues. Moreover, viewing the CD$\mathrm{ROM}$ prior to meeting with the genetic counselor may help the patient better understand the content areas that are subsequently presented by the genetic counselor during the session. More detail about the CD-ROM used in this study is provided in the Materials and Methods section.

The feedback checklist for the genetic counselor focused on providing the counselor with information on patients' prior knowledge of cancer and genetics. Feedback on prior knowledge and prior misconceptions may enable the genetic counselor to focus more time on the areas that are less understood by the patient and to spend less time on those areas that are well understood. This would allow the genetic counselor to personalize and tailor genetic counseling to the informational needs of the patients in an attempt to streamline the education component of the session, yet not at the expense of patient education and knowledge acquisition of difficult genetic concepts. Feedback information was based on a knowledge assessment that was completed by the patient prior to the start of the counseling appointment.

Evalutation of these strategies focused on relevant genetic counseling outcomes and included time spent face-to-face with the genetic team (i.e., both the genetic counselor and medical oncologist-timed separately), knowledge acquisition, alleviation of concerns (i.e., reducing worry), and actual genetic testing decisions. 
Women attending the Breast and Ovarian Cancer Risk Evaluation Program (BOCREP) at the University of Michigan Comprehensive Cancer Center were randomized to receive one of the following: (1) no pre-counseling education (standard care), (2) a CD-ROM to review before counseling, (3) feedback to the genetic counselor, or (4) CD-ROM to review and feedback to genetic counselor. Several hypotheses were tested in the present study:

- Women who view the CD-ROM will have better knowledge and understanding of genetic information than women who do not view the CD-ROM. They will also worry less about their risk and spend less time face-to-face with the genetics team.

- Feedback to counselor will result in improved patient knowledge, less worry and shorter face-to-face time with the genetics team.

- The combination of both CD-ROM and feedback to counselor strategies will produce a synergistic effect on knowledge, worry, and face-to-face time.

\section{MATERIALS AND METHODS}

\section{Subjects}

A total of 205 women attended the Breast and Ovarian Cancer Risk Evaluation Program (BOCREP) at the University of Michigan Comprehensive Cancer Center between November 2000 and December 2002. The BOCREP is a program that provides breast and ovarian cancer risk assessment, genetic counseling, and genetic testing for mutations in BRCA1 and $B R C A 2$. Women attending the BOCREP were either selfreferred or were referred by a physician or other health care provider due to concerns about risk for developing breast and/ or ovarian cancer. These women may be unaffected and have a family history of cancer, or may have had a previous cancer diagnosis. Clinic recommendations for genetic testing for BRCA1 and BRCA2 were based on a cutoff of $10 \%$ risk of familial mutation, where testing would be advised as reasonable [Couch et al., 1997; Frank, 1999]. If the chance of harboring a germline mutation in $B R C A 1 / 2$ was less than $10 \%$, testing was discouraged but not denied. Based on these criteria, the most appropriate candidate for initial testing was defined as follows: (1) self, (2) other family member (e.g., if the woman did not have a prior history of cancer), and (3) not encouraged to test (i.e., low risk).

All women attending the BOCREP were approached to take part in the randomized trial and eight women declined participation. A final total of 197 women are included in the present study.

\section{Measures}

Table I provides the study assessment time points.

Length of time in counseling. The total face-to-face counseling time spent with the genetic team was monitored and recorded for each session by a member of the research team.
Separate times were derived for time spent with the genetic counselor (KJM) and with the medical oncologist (SDM).

Knowledge. Knowledge was assessed using a 17-item multiple-choice instrument, developed at the University of Michigan (see Appendix A supplemental material, online). Items included questions about the causes of breast cancer, breast cancer genetics, genetic testing, and methods to reduce breast cancer risk (Cronbach's alpha $=0.72$ ).

Gene mutation worry. Worry about being a gene mutation carrier was assessed with a single item that asked, "As of this moment, how worried are you that you may have a gene mutation that may increase your risk for developing breast/ ovarian cancer?" Responses were rated on a 5-point Likert scale ranging from "not at all worried" to "extremely worried."

Genetic testing decisions. Testing decisions were recorded via a medical chart audit, approximately 1 year following the initial genetic counseling session.

\section{Procedures}

As part of the existing clinic protocol, all women contacting the clinic for an appointment are mailed an initial pre-clinic questionnaire package, which contains items assessing demographics, family history, and baseline psychosocial measures of interest. This initial questionnaire is to be completed at home and returned by mail to the clinic coordinator. An appointment is scheduled once the initial questionnaire is received.

Recruitment for the present study took place over the telephone when women contacted the clinic for an appointment. If a woman agreed to take part, she was asked to arrive at clinic 15 min prior to her scheduled appointment where she was greeted by a member of the research team $(\mathrm{CW})$ and asked to complete a knowledge assessment and informed consent. She was then randomly assigned to one of the four study conditions and spent the time prior to her appointment either by waiting or viewing the CD-ROM program. Those women in the CD-ROM condition were provided with brief instructions and a demonstration on how to use the program. Due to the nature of the clinic, the amount of time women had with the CD-ROM program varied and depended on when the genetic counselor was ready to see the patient. At a minimum, the women had 10-15 min to view at least one chapter in the program (of five). At maximum, they viewed the entire program (all five chapters). Unfortunately, the number of chapters (or parts of chapters) viewed was not recorded. Women in the CDROM condition were also informed that the genetic counselor was not aware of whether or not they viewed the CD-ROM. This was done in efforts to maximize the likelihood of the counselor remaining blind to the experimental condition.

If a woman was also randomized to an experimental condition in which the counselor was to receive feedback, her knowledge assessment was immediately coded and a feedback checklist was completed by a member of the research team (CW) and provided to the genetic counselor prior to the start of the session. The time spent with the genetic counselor and

TABLE I. Assessment Time Points

\begin{tabular}{lccccc}
\hline & \multicolumn{5}{c}{ Day of initial visit } \\
\cline { 2 - 6 } & Baseline & $\begin{array}{c}\text { Before } \\
\text { appointment }\end{array}$ & $\begin{array}{c}\text { During } \\
\text { appointment }\end{array}$ & $\begin{array}{c}\text { After } \\
\text { appointment } \\
\text { (exit) }\end{array}$ & $\begin{array}{c}\text { Chart } \\
\text { audit }\end{array}$ \\
\hline $\begin{array}{l}\text { Face-to-face time } \\
\begin{array}{l}\text { Worry about gene mutation } \\
\text { Knowledge }\end{array}\end{array}$ & $\mathrm{X}$ & & $\mathrm{X}$ & $\mathrm{X}$ & \\
\begin{tabular}{l} 
Genetic testing decision \\
\hline
\end{tabular} & & $\mathrm{X}$ & & $\mathrm{X}$ & $\mathrm{X}$ \\
\hline
\end{tabular}


medical oncologist was derived, based on when each entered (in-time) and exited (out-time) the counseling room. If interruptions occurred during counseling (rare), the exit time was adjusted for the time spent outside of the room.

All clinic sessions began with the genetic counselor who spent time reviewing information on the genetics of breast and ovarian cancer, inherited susceptibility (risk estimate) for cancer, and genetic testing if appropriate for the family. Recommendations were also made regarding cancer surveillance and diet and lifestyle behaviors. The medical oncologist met with patients after the genetic counselor to reiterate what was communicated by the genetic counselor and clarify any questions regarding medical management of risk. Women who chose to undergo genetic testing could either have blood drawn the day of the appointment or at a later date.

Immediately following genetic counseling, women were asked to complete an exit questionnaire, which contained a follow-up knowledge assessment and worry measure.

\section{Description of intervention strategies.}

$C D-R O M$. The CD-ROM used in this study is entitled "Understanding Cancer and Genetics" and was developed at the University of Michigan by the Breast and Ovarian Cancer Risk Evaluation Program clinic team and the Health Media Research Lab. This CD-ROM covers relevant information in five chapters: Basic Genetics, Cancer and Genetics, Genes Associated With Breast Cancer, Genetic Testing, and Managing Risk. In total, the CD-ROM takes approximately $40 \mathrm{~min}$ to review in its entirety. All patients randomized to the CD-ROM condition reviewed at least one of the introductory chapters: Basic Genetics or Cancer and Genetics. From there, those who had additional time could view any or all of the remaining chapters, or repeat any of the ones they had already seen. (For more information on the CD-ROM, please refer to the following websites: www.mi-cancergenetics.org or www.healthmedia. umich.edu/projects/cancergenetics.htm.)

Feedback to genetic counselor. In the feedback conditions, the genetic counselor was provided with a checklist detailing the areas on the knowledge assessment that were missed by the patient (see the online Appendix B at http://www. interscience.wiley.com/jpages/1552-4825/suppmat/index.html). Specific notes were made on the adjacent lines related to a content area to indicate how the patient misunderstood the area. For example, if a woman responded to item 11 on the knowledge assessment that the lifetime risk of developing breast cancer in the United States was one in two (50\%), rather than one in eight (12\%), a notation of 'one in eight, thinks risk is higher' was made on the first line of the feedback sheet and the first box was to be checked $(\checkmark)$. Or, if women incorrectly responded to item 4 on the knowledge assessment, which asks about the types of cancers males with a BRCA2 mutation are more likely to develop, the feedback was noted as 'male breast cancer' on the line beside the subheading "BRCA2 - associated cancers (women/men)." Feedback was consistent for most areas because the misconceptions were the same. However, in other circumstances, the feedback varied according to how the women misunderstood the area. For example, if a women responding to item 3 on the knowledge assessment only circled breast cancer as a response to the types of cancers associated with BRCA1 mutations, then a note 'ovarian cancer risk' was made. The opposite occurred if women circled only ovarian cancer, in which case 'breast cancer risk' was noted. Overall, any areas that were not checked on the feedback list suggested to the genetic counselor that the woman was correct in her response and understood the area.

\section{Analysis Plan}

The analysis was conducted in two stages. First, descriptive statistics were generated to describe the study population.
Two-way ANOVAs were performed to compare baseline characteristics across all groups. Next, a series of factorial ANOVAs were conducted. A number of $2 \times 2$ between-subjects factorial ANOVAs were conducted to determine the impact of the factors (CD-ROM/feedback) on face-to-face time, both combined and separate for the genetic counselor and medical oncologist. In addition, two $2 \times 2 \times 2$ factorial ANOVAs, with two factors between-subjects and one factor within-subjects, were conducted to determine the impact of the factors on changes in knowledge and worry following genetic counseling. Logistic regression was used to determine the impact of the factors on genetic testing decisions.

\section{RESULTS \\ Participation Bias}

A potential source of participation bias may stem from recruitment into the randomized trial. Of 205 women who attended clinic, eight $(3.9 \%)$ chose to not take part in the randomized trial. No differences were noted between those who accepted versus declined study participation on various demographic characteristics including age, education, actual breast cancer risk status, and worry about gene mutation.

\section{Characteristics of Study Population}

Baseline characteristics for the overall study population are presented in Table II. Study participants ranged in age from 22 to 76 years $(\mathrm{M}=45, \mathrm{SD}=10.3)$. The majority of women were Caucasian (93\%), married (80\%), reported having at least a bachelors degree or higher $(58 \%)$ and a household income greater than $\$ 60,000$ (66\%). A total of $30 \%$ of women had a prior history of breast (including DCIS) and/or ovarian cancer.

Eligibility for testing among the women was as follows: self $(51 \%)$, other family member $(32 \%)$, and not encouraged/low risk $(17 \%)$. Of the total sample, 79 of 197 women $(40 \%)$

TABLE II. Characteristics of Study Population

Characteristic \% of participants

Age, years

Mean $44-45$

Range $22-76$

Education

High school or less

Some college, no degree

Associates degree/vocational certificate

College graduate

Postgraduate degree

Ethnicity

Caucasian

$\%$ of participants

African American

Hispanic

Asian

Other

Marital status

Married

Single

Divorced

Other

Income

Less than $\$ 20,000$

$\$ 21,000-\$ 40,000-13$

$\$ 41,000-\$ 60,000 \quad 16$

Greater than $\$ 60,000 \quad 66$

Eligibility for testing

Self

Other family member

Not encouraged/low risk

\begin{tabular}{ll}
32 \\
\hline
\end{tabular}


underwent genetic testing. Among the subgroup of 100 women who were personally deemed appropriate for testing, testing rates were higher $(74 \%)$. Of those women who had a relative that was deemed the most appropriate candidate for testing, 4 of $63(6 \%)$ underwent genetic testing (with only one of the four having prior knowledge of a known mutation in the family). Only 1 individual of 34 (3\%) went ahead with genetic testing even though she was considered at low risk and not encouraged to test.

\section{Comparison of Experimental Groups at Baseline}

To determine whether the experimental conditions were comparable at baseline, two-way ANOVA calculations were performed to examine differences between groups on demographic characteristics (age, education, and prior history of cancer) and study variables of interest (knowledge, and worry). No baseline differences were noted for all main effects and interactions for any of the variables.

\section{Bivariate Associations Between Demographic Variables and Dependent Variables of Interest}

To determine whether any of the demographic variables may have influenced the impact of the randomized trial, we examined whether age, education, and prior history of cancer were associated with any of the dependent variables of interest. Age and education were dichotomized (less than age 50, 50+; less than bachelor's degree, bachelor's + ) in the present study. As such, correlations reported are point biserial correlations. No differences were noted when examining age as a continuous variable.

Age was significantly associated with the amount of time the genetic counselor spent with the patient $(r=0.15, P<0.05)$, such that the genetic counselor spent more time face-to-face with older patients $(\mathrm{M}=48 \mathrm{~min}, \mathrm{SD}=15)$ than younger patients $(\mathrm{M}=44 \mathrm{~min}, \mathrm{SD}=12)$. In contrast, education was significantly associated with the amount of time the medical oncologist spent with the patient $(\mathrm{r}=0.15, P<0.05)$, such that the medical oncologist spent more time with those with a bachelor's degree or higher $(\mathrm{M}=17 \mathrm{~min}, \mathrm{SD}=9)$ compared to those with less than a bachelor's degree $(\mathrm{M}=15 \mathrm{~min}, \mathrm{SD}=8)$. Education was positively associated with post-counseling knowledge $(\mathrm{r}=0.18, P=0.01)$ and marginally associated with lower worry at follow-up $(\mathrm{r}=-0.14, P=0.06)$. Neither age nor education was associated with genetic testing decisions. Prior history of cancer was significantly associated with genetic testing decisions $(r=0.48, P<0.001)$, such that those women with a prior history were more likely to undergo genetic testing. Cancer history was not associated with any other dependent variables of interest. In light of these findings, all subsequent analyses involving time, knowledge, and worry variables included both age and education as covariates. Analyses pertaining to genetic testing decisions controlled for prior history of cancer.

\section{Impact of Randomized Trial on Face-to-Face Time}

Face-to-face time was recorded separately for the genetic counselor and the medical oncologist. As such, a series of 2 (CD) $\times 2$ (feedback to counselor) factorial ANOVAs were conducted to determine whether the strategies had an impact on reducing the amount of time spent with the genetic counselor alone, with the medical oncologist alone, and with both combined. Overall, the time spent with the genetic counselor was strongly associated with the time spent with the medical oncologist $(\mathrm{r}=0.42, P<0.001)$. Table III provides a summary of face-to-face time.

There was a main effect of the CD-ROM on time spent faceto-face with the genetic counselor, such that women who
TABLE III. Face-to-Face Time (in minutes), Adjusted for Age and Education

\begin{tabular}{lcccc}
\hline & $\begin{array}{c}\text { Control } \\
(\mathrm{n}=48)\end{array}$ & $\begin{array}{c}\text { CD } \\
(\mathrm{n}=50)\end{array}$ & $\begin{array}{c}\text { Feed } \\
(\mathrm{n}=49)\end{array}$ & $\begin{array}{c}\text { CD + Feed } \\
(\mathrm{n}=50)\end{array}$ \\
\hline $\begin{array}{l}\text { Counselor only } \\
\text { M }\end{array}$ & $0: 49$ & $0: 43$ & $0: 47$ & $0: 44$ \\
$\quad$ SD & $(0: 12)$ & $(0: 12)$ & $(0: 13)$ & $(0: 13)$ \\
$\begin{array}{l}\text { Medical oncologist only } \\
\quad \text { M }\end{array}$ & $0: 19$ & $0: 16$ & $0: 14$ & $0: 16$ \\
$\quad$ SD & $(0: 08)$ & $(0: 08)$ & $(0: 08)$ & $(0: 08)$ \\
$\begin{array}{l}\text { Both combined } \\
\quad \text { M }\end{array}$ & $0: 68$ & $0: 59$ & $0: 61$ & $0: 60$ \\
$\quad$ SD & $(0: 17)$ & $(0: 17)$ & $(0: 17)$ & $(0: 17)$ \\
\hline
\end{tabular}

viewed the CD-ROM spent significantly less time with the counselor compared to women who did not view the CD-ROM, $\mathrm{F}(1,189)=6.35, P=0.01$. Neither feedback to counselor nor the interaction of the two strategies had a significant impact on time spent with the genetic counselor.

In contrast, there was a main effect of the feedback to counselor strategy on time spent with the medical oncologist. Women randomized to the feedback condition spent significantly less time with the medical oncologist compared to women who were not, $\mathrm{F}(1,188)=5.42, P<0.05$. Neither the main effect for the CD-ROM condition nor the interaction between the two strategies was significant.

Combined time with both the genetic counselor and medical oncologist varied as a function of the CD-ROM condition, with less total face-to-face time spent with women who viewed the CD-ROM, F $(1,188)=4.09, P<0.05$. Neither the main effect for feedback nor the interaction between CD-ROM and feedback was significant.

\section{Changes in Knowledge and Worry by Experimental Group}

Knowledge. Two $2(\mathrm{CD}) \times 2$ (feedback) $\times 2$ (time) factorial ANOVAs were conducted to determine the impact of the randomized trial on changes in both knowledge and worry over time. Overall, knowledge significantly improved from baseline to follow-up, $\mathrm{F}(1,185)=4.78, P<0.05 \quad\left(\mathrm{M}_{\text {baseline }}=11.48\right.$, $\left.\mathrm{SD}=2.86 ; \mathrm{M}_{\text {follow-up }}=13.02, \mathrm{SD}=3.18\right)$. More importantly, this improvement varied by feedback strategy (see Fig. 1). Women who were randomized to the feedback condition experienced significantly greater gains in knowledge $\left(\mathrm{M}_{\text {baseline }}=\right.$ 11.36, $\mathrm{SD}=2.84 ; \mathrm{M}_{\text {follow-up }}=13.40, \mathrm{SD}=3.16$ ) compared to women not randomized to the feedback condition, $\mathrm{F}(1,185)=$

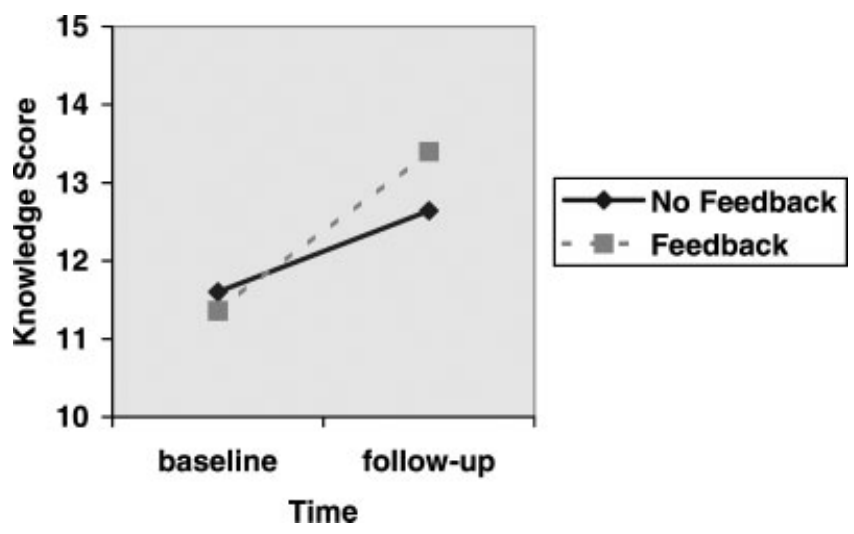

Fig. 1. Changes in knowledge over time by feedback 
3.96, $P<0.05\left(\mathrm{M}_{\text {baseline }}=11.60, \mathrm{SD}=2.86 ; \mathrm{M}_{\text {follow-up }}=12.64\right.$, $\mathrm{SD}=3.18)$

Worry. Worry about having a gene mutation significantly declined from baseline to follow-up, $\mathrm{F}(1,177)=3.78, P=0.05$ $\left(\mathrm{M}_{\text {baseline }}=3.47, \mathrm{SD}=1.10 ; \mathrm{M}_{\text {follow-up }}=3.00, \mathrm{SD}=1.03\right)$. This decline may have varied as a function of the CD-ROM, as suggested by the trend towards a main effect noted for this strategy. That is, women who viewed the CD-ROM experienced slightly greater declines in worry from baseline to follow-up, compared to women who did not view the CD-ROM, F (1, $177)=3.51, P=0.06$. Neither feedback to counselor nor the interaction between $\mathrm{CD}-\mathrm{ROM}$ and feedback appeared to influence the changes in worry over time.

Post-hoc analyses were conducted in attempts to better understand the possible impact of the CD-ROM on change in worry over time. More specifically, two additional 2 (CD) $\times 2$ (feedback) $\times 2$ (time) factorial ANOVAs were conducted, stratifying by baseline worry (median split, $<4$ vs. $\geq 4$ of 5 ). Among women high in baseline worry, no differences were noted between CD-ROM viewers and non-viewers in the changes in worry following counseling. In contrast, changes in worry over time among women low in baseline worry did vary as a function of the CD-ROM. As shown in Figure 2, women who viewed the CD-ROM did not change in worry over time $\left(\mathrm{M}_{\text {baseline }}=2.57, \mathrm{SD}=0.70 ; \mathrm{M}_{\text {follow-up }}=2.46, \mathrm{SD}=0.97\right)$, in contrast to women who did not view the CD-ROM who experienced an increase in worry following genetic counseling, $\mathrm{F}(1,82)=10.02, P<0.005\left(\mathrm{M}_{\text {baseline }}=2.40, \mathrm{SD}=0.70\right.$; $\left.\mathrm{M}_{\text {follow-up }}=3.02, \mathrm{SD}=0.98\right)$.

\section{Impact of Randomized Trial on the Genetic Testing Decision}

A logistic regression was run to determine whether there were any differences in the proportion of women choosing to undergo genetic testing by experimental group. There was a significant main effect for the CD-ROM on genetic testing decisions: specifically, women who viewed the CD-ROM were significantly less likely to undergo genetic testing compared to women who did not view the CD-ROM ( $33 \%$ vs. $47 \%$; OR $=0.63$, $\mathrm{CI}=[0.45-0.89], P<0.01)$. No differences were noted in testing decisions by feedback to the counselor or CD$\mathrm{ROM} \times$ feedback. These findings remained consistent even when re-analyzed with the subgroup of women who were deemed as the most appropriate person for genetic testing, where there are fewer barriers to testing such as an affected relative unwilling to undergo testing. As expected, overall testing rates were higher among this subgroup and women who viewed the CD-ROM were significantly less likely to undergo genetic testing compared to women who did not view the CD$\mathrm{ROM}(64 \%$ vs. $83 \%$; $\mathrm{OR}=0.56, \mathrm{CI}=[0.34-0.92], P<0.05)$.

\section{DISCUSSION}

With the increasing demand for genetic counseling services comes the need to create new stategies to assist in the counseling process. Our study set out to determine the efficacy of two strategies to facilitate genetic counseling for $B R C A 1 / 2$, each focusing on a different intervention target. Not only was it important to consider how these strategies could help genetics professionals save time, it was also critical to determine how they could affect indicators of counseling success. Moreover, the intent of these strategies was not to replace already existing services, but to enhance them. As such, the goal here was not to establish whether a CD-ROM was more or less effective at educating patients than genetic counselors, but to determine whether it would be worthwhile to include this strategy (and others) to compliment the education and counseling already being provided.

Overall, the CD-ROM reduced the amount of time spent faceto-face with the genetic counselor. It did not, however, have an influence on improving knowledge acquisition. Although the CD-ROM did not result in better knowledge acquisition, it did result in the same knowledge gains over a shorter time period. In other words, the shorter time spent face-to-face with the genetic counselor was not at the expense of patient education. This outcome replicates the findings by others that multimedia educational aids, such as a CD-ROM computer program, can be useful for educating patients on cancer and genetics [Green et al., 2001a, 2004]. In turn, such programs can help reduce the burden on genetic counselors, allowing them to focus on other issues that may be important during the encounter.

The CD-ROM also had an impact on worry about having a gene mutation for those women who were less worried at baseline. Among this subgroup, women who did not view the CD-ROM showed a significant increase in worry over time, compared to women who saw the CD-ROM who did not change in their worry. Several possible explanations may account for this finding. Women who did not view the CD-ROM may have become more overwhelmed with all the information conveyed during genetic counseling, which resulted in the increase in worry. Also, viewing the CD-ROM may have enabled the genetic counselor to spend more time on psychosocial issues pertaining to testing, which may have prevented an increase in worry. Interestingly, CD-ROM viewers were significantly less likely to undergo genetic testing compared to nonviewers. Because genetic testing uptake was also found to be significantly associated with worry at follow-up $(\mathrm{r}=.26, P<0.001)$,

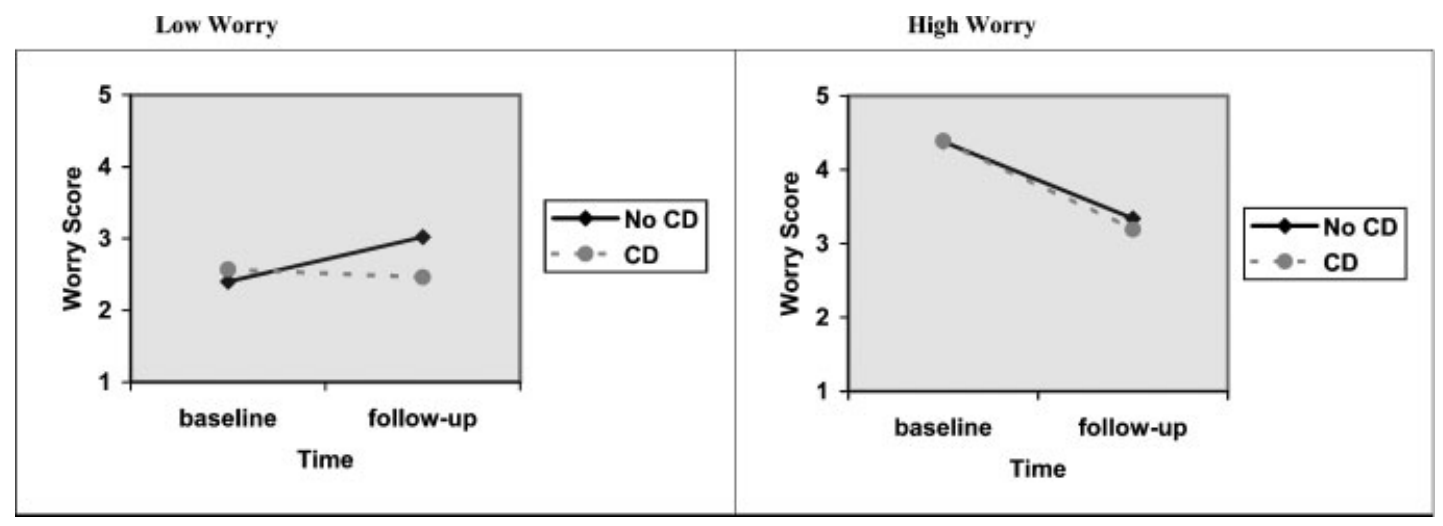

Fig. 2. Changes in worry over time by CD-ROM, stratified by baseline worry. 
the observed increase in worry among nonviewers raises concerns about the extent to which the increase may have resulted in more women choosing to have genetic testing.

The difference in genetic testing uptake between CD-ROM viewers and nonviewers may be due to several explanations. First, as already mentioned, the genetic counselor may have been able to better address and alleviate the psychosocial concerns of the women who had viewed the CD-ROM. Second, those who saw the CD-ROM may have been exposed to the chapter on genetic testing, which systematically reviews the benefits, risks, and limitations of genetic testing. As such, women who viewed the CD-ROM may have had shifts in their perceived benefits or barriers to testing, both of which have been deemed important for testing decisions [Lerman et al., 1996; Jacobsen et al., 1997; Cappelli et al., 1999; Bosompra et al., 2000]. Lerman et al. [1997] also found that counseling interventions can decrease perceived benefits of testing, however, their study did not demonstrate an impact on testing intentions. This is in contrast to others who have generally found interest in genetic testing to decline following genetic counseling [Burke et al., 2000; Green et al., 2001a, 2004], which may be attributed to an increased understanding of the limitations of testing, especially among women who are not deemed at high risk for hereditary breast-ovarian cancer. Contrary to the findings by Green et al. [2004], where testing decisions did not differ between viewers and nonviewers of a CDROM, our study is the first to demonstrate an impact of a CDROM supplement in reducing genetic testing uptake. A greater understanding of why genetic testing declines is essential to ensure that women are making informed decisions about genetic testing. Future research should examine the potential value of interactive multimedia applications to facilitate decision-making for genetic testing in additional samples.

Feedback to the genetic counselor did not result in shorter consultation times with the counselor: this may be attributed to how she chose to spend her time with patients. For example, if a patient was already very knowledgeable about cancer and genetics, the genetic counselor may have spent her time covering more psychosocial related issues. In contrast, if the patient did not have a good understanding of cancer and genetics, then the time may have been spent reviewing this information to ensure better understanding. If this were the case, then the counselor would have spent the same amount of time with patients regardless of what they knew coming into the counseling session. Although we did not record how the genetic counselor spent her time during the session, the lack of association between baseline patients' baseline knowledge and face-to-face time with the genetic counselor adds some support to this speculation.

In contrast, feedback to the genetic counselor did result in shorter consultation times with the medical oncologist. As this study also demonstrated, the feedback to counselor strategy resulted in greater gains in knowledge, suggesting that this process may subseqently place less demands on the medical oncologist's time. It should be noted that actual knowledge at follow-up was not predictive of time spent with the medical oncologist. As such, other factors may account for the differences noted. It is possible that women in the feedback to counselor condition experienced more personalized counseling that better met their needs and resulted in fewer issues that the medical oncologist needed to address. Another possibility is that the genetic counselor may have interacted differently with the medical oncologist during the consultation immediately following the counselor's session with the patient. Although the medical oncologist was blind to the feedback condition, it is possible the genetic counselor provided different information about those patients whom she received feedback, which resulted in the shorter consultation times with the medical oncologist.
The impact of the feedback strategy on knowledge acquisition was consistent with what we anticipated. This finding also adds support to prior evidence of the benefits of tailoring genetic information on outcomes such as knowledge and risk comprehension [Skinner et al., 2002]. As such, this finding suggests that there is merit to providing counselors with additional information on their patients' baseline understanding of cancer and genetics to facilitate the counseling encounter. The relatively simple approach of providing a checklist to enhance counseling has important implications on strategies employed to enable informed decision making. Future research may benefit from examining other information that could be included on a feedback checklist that may help counselors to better personalize and tailor their sessions to meet the needs of their clients. Moreover, researchers should also continue to explore the benefits of providing tailored feedback directly to patients. A better understanding of how to best integrate tailoring strategies into the genetic counseling encounter will also be critical. As issues pertaining to lifestyle modification increasingly enter into the genetic counseling realm [Marteau and Lerman, 2001], tailored approaches, and their demonstrated impact on health behaviors [see Skinner et al., 1994; Brug et al., 1999; Bull et al., 1999; Strecher, 1999], may provide the tools needed to meet these challenges.

Our study did not demonstrate any synergistic effects for the two strategies. It is possible that the two strategies work on different processes important for various outcomes of interest. More specifically, the processes involved in knowledge acquisition may differ from the processes involved in decision making. For example, decision making may be more influenced by a detailed review of the benefits, risks, and limitations associated with each option (i.e., to test or not) rather than by knowledge of key genetic concepts. The CDROM may have provided a more detailed review of benefits and risks of genetic testing, above and beyond the feedback to counselor strategy, which focused more specifically on knowledge misconceptions. This may account for why synergistic effects were not noted on outcomes such as genetic testing decisions, for which the CD-ROM had an impact. Future multimedia applications may benefit from a combined approach of a computer education program that also incorporates tailored feedback to the patient.

A primary strength of this study is the examination of actual genetic testing decisions, rather than testing intentions, which are not always reflective of behavior [Cappelli et al., 1999; Croyle and Lerman, 1999]. Genetic testing decisions were obtained via medical chart audit approximately 1 year following the initial genetic counseling session to allow for the time needed to account for possible delays in genetic testing. These can include seeking and arranging health or life insurance, talking to family members about testing (especially if they are better candidates), or personal uncertainty about the decision to test. Although the study design enabled us to potentially account for barriers that may delay testing uptake, we are unable to explain changes in decisions over this time frame. For example, women who initially decide not to test but change their minds a few months later may have various reasons for the decision reversal (e.g., increase in worry after a new diagnosis in the family). Future research should examine the process of decision making over time to better understand the important predictors of genetic testing decisions.

Our study participants were predominantly Caucasian and well-educated. As such, our findings are limited in generalizability to similar populations and should be interpreted accordingly. Generalizability of the study findings may also be limited as all patients saw the same genetic counselor and medical oncologist. As such, we cannot examine how different clinicians' personal counseling style may have influenced the study outcomes. 
Although efforts were made to keep the genetics team blind to study conditions, we cannot guarantee that women who viewed the CD-ROM did not interact with the counselor in ways that would disclose the study condition. Future studies would possibly benefit from randomly taping genetic counseling sessions to check the content of the sessions.

Another study limitation pertained to not knowing the content of what patients viewed when they saw the CD-ROM. Because this information was not collected, our study could not address questions related to dose response effects nor chapter/ content effects. Although we could have designed the study to have patients view the entire CD-ROM prior to genetic counseling, this would have been too disruptive for the clinic setting. Moreover, because we were trying to integrate the CDROM as smoothly as possible into the clinic, we felt it best to have the limitations of a real life setting take precedence in order to maximize the practical application of this approach. Nonetheless, due to the structure of many clinical settings, future research would also benefit from identifying ways to provide the information contained on the CD-ROM to women before their appointment. This could entail either mailing the CD-ROM to women or providing the content over the Internet. These alternate approaches outside of clinic would also allow women to view the information within the constraints of their personal schedule and reduce the burden of time that they would otherwise spend in clinic viewing the information.

Despite these limitations, our study has demonstrated the potential usefulness of various strategies that can focus on different intervention targets to facilitate the counseling process. Further insights into the mechanisms by which these strategies influence desired counseling outcomes is imperative.

\section{REFERENCES}

Barrios LC, Runyan CW, Downs SM, Bowling JM. 2001. Pediatric injury prevention counseling: An observational study of process and content. Patient Educ Couns 44:141-149.

Bordley WC, Margolis PA, Stuart J, Lannon C, Keyes L. 2001. Improving preventive service delivery through office systems. Pediatrics 108(3): e41.

Bosompra K, Flynn BS, Ashikaga T, Rairikar CJ, Worden JK, Solomon LJ. 2000. Likelihood of undergoing genetic testing for cancer risk: A population-based study. Prev Med 30:155-166.

Brug J, Campbell M, van Assema P. 1999. The application and impact of computer-generated personalized nutrition education: A review of the literature. Patient Educ Couns 36:145-156.

Bull FC, Kreuter MW, Scharff DP. 1999. Effects of tailored, personalized and general health messages on physical activity. Patient Educ Couns $36: 181-192$

Burke W, Culver JO, Bowen D, Lowry D, Durfy S, McTiernan A, Andersen MR. 2000. Genetic counseling for women with an intermediate family history of breast cancer. Am J Med Genet 90:361-368.

Cappelli M, Surh L, Humphreys L, Verma S, Logan D, Hunter A, Allanson J. 1999. Psychological and social determinants of women's decisions to undergo genetic counseling and testing for breast cancer. Clin Genet $55: 419-430$

Couch FJ, DeShano ML, Blackwood A, Calzone K, Stopfer J, Campeau L, Ganguly A, Rebbeck T, Weber BL. 1997. BRCA1 mutations in women attending clinics that evaluate the risk of breast cancer. N Engl J Med 336:1409-1415.

Croyle RT, Lerman C. 1999. Risk communication in genetic testing for cancer susceptibility. J Natl Cancer Inst Monogr 25:59-66.

Cull A, Miller H, Porterfield T, Mackay J, Anderson EDC, Steel CM, Elton RA. 1998. The use of videotaped information in cancer genetic counseling: A randomized evaluation study. Br J Cancer 77(5):830-837.

Dickinson JC, Warshaw GA, Gehlbach SH, Bobula JA, Muhlbaier LH, Pakerson GR Jr. 1981. Improving hypertension control: Impact of computer feedback and physician education. Med Care 19(8):843-854.

Engelman K, Ellerbeck E, Perpich D, Nazir N, McCarter K, Ahluwalia J. 2004. Office systems and their influence on mammography use in rural and urban primary care. J Rural Health 20(1):36-42.
Esplen MJ, Toner B, Hunter J, Glendon G, Butler K, Field B. 1998. A group therapy approach to facilitate integration of risk information for women at risk for breast cancer. Can J Psychiatry 43(4):375-380.

Esplen MJ, Toner B, Hunter J, Glendon G, Liede A, Narod S, Stuckless N, Butler K, Field B. 2000. A supportive-expressive group intervention for women with a family history of breast cancer: Results of a phase II study. Psycho-Oncology 9:243-252.

Frank TS. 1999. Testing of hereditary risk for ovarian cancer. Cancer Control 6:327-334.

Geller G, Botkin JR, Green MJ, Press N, Biesecker BB, Wilfond B, Grana G Daly MB, Schneider K, Kahn MJE. 1997. Genetic testing for susceptibility to adult-onset cancer: The process and content of informed consent. JAMA 277(18):1467-1474.

Green MJ, Fost N. 1997. Who should provide genetic education prior to gene testing? Computers and other methods for improving patient understanding. Genetic Testing 1(2):131-136.

Green MJ, Biesecker BB, McInerney AM, Mauger D, Fost N. 2001a. An interactive computer program can effectively educate patients about genetic testing for breast cancer susceptibility. Am J Med Genet 103: 16-23.

Green MJ, McInerney AM, Biesecker BB, Fost N. 2001b. Education about genetic testing for breast cancer susceptibility: Patient preferences for a computer program or genetic counselor. Am J Med Genet 103: 24-31.

Green MJ, Peterson SK, Baker MW, Harper GR, Friedman LC, Rubinstein WS, Mauger DT. 2004. Effect of a computer-based decision aid on knowledge, perceptions, and intentions about genetic testing for breast cancer susceptibility. JAMA 292(4):442-452.

Guttmacher AE, Jenkins J, Uhlmann WR. 2001. Genomic medicine: Who will practice it? A call to open arms. Am J Med Genet 106:216-222.

Jacobsen PB, Valdimarsdottir HB, Brown KL, Offit K. 1997. Decisionmaking about genetic testing among women at familial risk for breast cancer. Psychosom Med 59:459-466.

Lane DS, Messina CR, Grimson R. 2001. An educational approach to improving physician breast cancer screening practices and counseling skills. Patient Educ Couns 43:287-299.

Lerman C, Narod S, Schulman K, Hughes C, Gomez-Caminero A, Bonney G, Gold K, Trock B, Main D, Lynch J, et al. 1996. BRCA1 testing in families with hereditary breast-ovarian cancer: A prospective study of patient decision making and outcomes. JAMA 275(24):1885-1892.

Lerman C, Biesecker B, Benkendorf JL, Kerner J, Gomez-Caminero A Hughes C, Reed MM. 1997. Controlled trial of pretest education approaches to enhance informed decision-making for BRCA1 gene testing. J Natl Cancer Inst 89(2):148-157.

Marteau TM, Lerman C. 2001. Genetic risk and behavioural change. Br Med $\mathrm{J}$ (Clin Res Ed) 322:1056-1059.

Miki Y, Swensen J, Shattuck-Eidens D, Futreal PA, Harshman K, Tavtigian S, Liu Q, et al. 1994. A strong candidate for the breast and ovarian cancer susceptibility gene BRCA1. Science 266:66-75.

Schneider KA. 1997. Genetic counseling for BRCA1/BRCA2 testing. Genetic Testing 1(2):91-98.

Schneider KA, Marnane D. 1997. Cancer risk counseling: How is it different? Journal of Genetic Counseling 6(2):97-109.

Skinner CS, Strecher VJ, Hospers H. 1994. Physicians' recommendations for mammography: Do tailored messages make a difference? Am J Public Health 84(1):43-49.

Skinner CS, Schildkraut JM, Berry D, Calingaert B, Marcom PK, Sugerman J, Winer EP, Iglehart JD, Futreal PA, Rimer BK. 2002. Pre-counseling education materials for BRCA testing: Does tailoring make a difference? Genetic Testing 6(2):93-105.

Strecher VJ. 1999. Computer-tailored smoking cessation materials: A review and discussion. Patient Educ Couns 36:107-117.

Tessaro I, Borstelmann N, Regan K, Rimer BK, Winer E. 1997. Genetic testing for susceptibility to breast cancer: Findings from women's focus groups. J Women's Health 6(3):317-327.

Tierney WM, Hui SL, McDonald CJ. 1986. Delayed feedback of physician performance versus immediate reminders to perform preventive care: Effects on physician compliance. Med Care 24(8):659-666.

Wang C, Gonzalez R, Merajver SD. 2004. Assessment of genetic testing and related counseling services: Current research and future directions. Soc Sci Med 58:1427-1442.

Wooster R, Bignell G, Lancaster J, Swift S, Seal S, Mangion J, et al. 1995 Identification of the breast cancer gene BRCA2. Nature 378:789-791. 